\title{
Acyclic orientations do not lead to optimal deadlock-free packet routing algorithms
}

\author{
Daniel Štefankovič ${ }^{1}$ \\ Department of Computer Science, Comenius University, Bratislava, Slovakia
}

\begin{abstract}
In this paper we consider the problem of designing deadlock-free shortest-path routing algorithms. A design technique based on acyclic orientations has proven to be useful for many important topologies, e.g., meshes, tori, trees and hypercubes. It was not known whether this technique always leads to algorithms using an asymptotically optimal number of buffers. We show this is not the case by presenting a graph of size $\mathcal{N}$ which has a deadlock-free shortest-path routing algorithm using $\mathrm{O}(1)$ buffers, but every deadlock-free shortest-path routing algorithm based on acyclic orientations requires $\Omega(\log \mathcal{N} / \log \log \mathcal{N})$ buffers. ㄷ 2000 Elsevier Science B.V. All rights reserved.
\end{abstract}

Keywords: Deadlock-free packet routing; Acyclic orientations; Interconnection networks

\section{Introduction}

Devising deadlock-free routing algorithms is an important problem in the network design. A wide range of different algorithms and design techniques has been proposed [1,4-11]. In this paper we concentrate on an important class of deadlock-free routing algorithms called buffer-reservation algorithms [2, 3]. Given source, destination and current buffer of a packet a buffer-reservation algorithm specifies a set of buffers to which the packet may move. The packet can move to any buffer from the specified set provided that it is empty.

It is known that for each network there exists a deadlock-free routing algorithm using only 2 buffers

\footnotetext{
This research has been partially supported by VEGA 1/4315/97.

${ }^{1}$ Email: stefanko@zeus.dcs.fmph.uniba.sk.
}

per node [8]. However this algorithm suffers from congestion, because it uses only a spanning tree of the network. It is reasonable to consider routing algorithms using only shortest paths. For such algorithms it was shown in [2] that there exists a network of size $\mathcal{N}$ which requires $\Omega(\log \log \mathcal{N})$ buffers per node. It is also known that for every network of diameter $\mathcal{D}$ there exists a shortest-path deadlock-free routing algorithm using $\mathcal{D}+1$ buffers per node [9].

A technique for constructing deadlock-free routing algorithms based on acyclic orientations has proven to be useful for many important topologies [9]. Moreover only few additional bits of data besides routing information are required by the algorithms designed using this technique. Thus a natural question is how good the technique is in general. We present a graph of size $\mathcal{N}$ which has deadlock-free shortest-path routing algorithm using $\mathrm{O}(1)$ buffers per node, but every deadlock-free shortest-path routing algorithm based 
on acyclic orientations requires $\Omega(\log \mathcal{N} / \log \log \mathcal{N})$ buffers per node. The proofs presented in this paper can be adapted to obtain the same result for the cubeconnected-cycles graph.

\section{Preliminaries}

A communication network is modeled by an undirected graph $G=(V, E)$, where vertices in $V$ represent nodes and edges in $E$ represent bidirectional communication links. Every edge is considered to comprise two opositely oriented arcs.

Given source, destination and the buffer currently holding the packet a buffer-reservation algorithm specifies a set of buffers to which the packet may move. The packet can move to any of the specified buffers, provided it is empty. A deadlock is a situation in which a set of packets can never reach their destination, because specified buffers of each packet are occupied by other packets from the set. A buffer reservation algorithm is called deadlock-free if it does not allow a deadlock to occur. A routing algorithm is called shortest path if the packets are always routed along shortest paths.

Given a graph $G$ an all-to-all path system $\mathcal{P}$ is a collection of paths connecting every two vertices in $G$. An orientation $D G$ of $G$ is a directed graph obtained from $G$ by replacing each undirected edge in $G$ by an arc (i.e., $\{u, v\}$ is replaced by either $(u, v)$ or $(v, u))$. An orientation is called acyclic if it doesn't contain a cycle. An orientation cover of a path system $\mathcal{P}$ is a sequence of orientations $D G_{1}, \ldots, D G_{s}$ such that every path $p \in \mathcal{P}$ can be written as a concatenation of $s$ paths $p_{1}, \ldots, p_{s}$ where $p_{i}$ is a path in $D G_{i}$ for $i \in[s]$. An acyclic orientation cover is an orientation cover consisting of acyclic orientations.

Let $\mathcal{P}$ be a shortest-path all-to-all path system of a graph $G$. If an acyclic orientation cover for $\mathcal{P}$ of size $s$ exists, then there exists a shortest-path deadlock-free routing algorithm using only $s$ buffers per vertex [9]. A routing algorithm designed using this technique is said to be based on acyclic orientations. If there is no restriction on how a routing algorithm was designed then it is said to use plain buffers.

\section{A gap between acyclic orientations and plain buffers}

In this section we present a graph which has shortest-path deadlock-free routing algorithm using only $\mathrm{O}(1)$ plain buffers per vertex, but every shortestpath deadlock-free routing algorithm based on acyclic orientations requires $\Omega(\log \mathcal{N} / \log \log \mathcal{N})$ buffers per vertex.

Consider the following machine $M_{n}$ :



It has a working tape with $n$ cells and a head which can be positioned above any cell. Each cell contains one binary digit. In one step the head can change the content of the scanned cell (shuffle) or it can move to the left or to the right neighboring cell. The state of the machine $M_{n}$ is the position of the head and the content of the tape.

Definition 1. Let $G_{n}$ be the graph with vertices corresponding to states of the machine $M_{n}$ and edges connecting two vertices iff the corresponding states are reachable in one step of $M_{n}$.

Remark 2. If the tape of the machine was wraparound the resulting graph would be the cube-connectedcycles graph. It is possible to show the same results for this graph with slightly more complicated proofs and worse constants.

\subsection{Upper bound for plain buffers}

Theorem 3. There exists a shortest-path deadlockfree routing algorithm of the graph $G_{n}$ using only 8 buffers per vertex.

Proof. Let $u, v$ be any two vertices of the graph $G_{n}$. Moving along a shortest path from $u$ to $v$ corresponds to changing the state $u$ to the state $v$ using the minimal number of steps. Let $h_{u}$ and $h_{v}$ be positions of the head in $u$ and $v$. Moreover let $l_{u, v}$ and $r_{u, v}$ be positions of the leftmost and the rightmost cell that have to be visited (every cell in which tapes of $u$ and $v$ differ as well as cells at positions $h_{u}$ and $h_{v}$ must be visited). 
In every shortest path the head makes a movement in one of the following two forms:

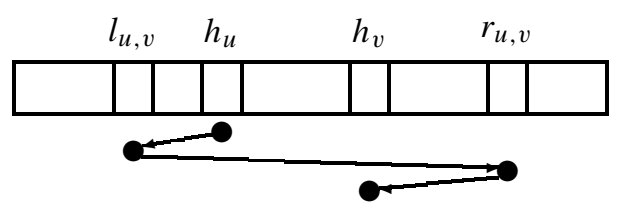

CASE $1\left(h_{u} \leqslant h_{v}\right)$

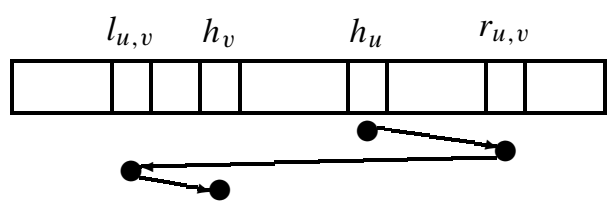

CASE $2\left(h_{u} \geqslant h_{v}\right)$

Thus according to the head movement each path can be divided into three phases (the first and/or third phase can be empty) in which the head moves in one direction. Now we show that eight buffers per vertex suffice. Each vertex has buffers with names $1,2,3,4,1^{\prime}, 2^{\prime}, 3^{\prime}, 4^{\prime}$. A packet moving along a path with the head movement-CASE 1 -is initially put in the buffer 1 and then it moves between buffers according to these rules:

- left-arc (phase 1)

- right-arc (phase 2)

any buffer $\rightarrow$ buffer 1

- left-arc (phase 3) any buffer $\rightarrow$ buffer 2

- shuffle-arc (all phases) any buffer $\rightarrow$ buffer 3

buffer $a \rightarrow$ buffer $a^{\prime}$

No shortest path contains two consecutive shuffleedges and thus the rule for the shuffle-arc is correct (a packet that wants to move along a shuffle-arc cannot be in a buffer with a prime). A packet moving along a path with the head movement-CASE 2-is initially put in buffer 2 and moves between buffers according to these rules:

- right-arc (phase 1)

any buffer $\rightarrow$ buffer 2

- left-arc (phase 2) any buffer $\rightarrow$ buffer 3

- right-arc (phase 3) any buffer $\rightarrow$ buffer 4

- shuffle-arc (all phases) buffer $a \rightarrow$ buffer $a^{\prime}$

For the sake of contradiction suppose that there is a deadlock. Then there must be a sequence such that

$\left(v_{1}, b_{1}\right) \rightarrow\left(v_{2}, b_{2}\right) \rightarrow \cdots \rightarrow\left(v_{m}, b_{m}\right) \rightarrow\left(v_{1}, b_{1}\right)$,

where $(u, a) \rightarrow(v, b)$ means that a packet from the buffer $a$ in the vertex $u$ waits for the buffer $b$ in the vertex $v$. Observe that a packet never moves to a buffer with smaller number. Therefore all buffers $b_{1}, \ldots, b_{m}$ must have the same number-some $a \in$ [4]. When a packet moves to a buffer $a^{\prime}$ it uses shuffle-arc and when it moves to a buffer $a$ it uses a left-arc if $a$ is odd and a right-arc if $a$ is even. Therefore all buffers $b_{1}, \ldots, b_{m}$ must be $a^{\prime}$. However a packet never moves from $a^{\prime}$ to $a^{\prime}$, a contradiction.

\subsection{Lower bound for acyclic orientations}

The vertex set of an $n$-dimensional hypercube consists of all binary strings of length $n$. Two vertices are connected by an edge iff they differ in exactly one bit. For any two vertices $u, v$ a movement along path from $u$ to $v$ corresponds to a sequence of changes of some bits. If the bits are changed in order from left to right then the path is called monotone. The lower bound for acyclic orientations depends on the following lemma:

Lemma 4. Let $\mathcal{P}$ be the all-to-all path system of an n-dimensional hypercube with monotone paths. Every orientation cover for $\mathcal{P}$ has size $\Omega(n / \log n)$.

Proof. Let $D G_{1}, \ldots, D G_{s}$ be an orientation cover for $\mathcal{P}$. We show that the size of this cover must be large by simulating movement of packets in the network. Each vertex generates a packet for every other vertex. The packets then move along their paths according to the orientation $D G_{1}$, then according to $D G_{2}$ and so on and finally after moving according to $D G_{s}$ every packet must be in its destination. We observe the state of the network (positions of packets) after each orientation.

Let $d$ be a number $0 \leqslant d \leqslant n$ and $v$ be a vertex. By $S_{v}^{d}$ we denote the set of all vertices that can differ from $v$ only in the first $d$ bits and by $D_{v}^{d}$ the set of all vertices that can differ from $v$ only in the last $n-d$ bits. Given a state of the network we call the vertex $v$ active with respect to $d$ if for each vertex $u \in D_{v}^{d}$ there exists a packet which has to move to $u$ through $v$. Clearly if $v$ has not yet received some packet $\langle p\rangle$ from some vertex $w \in S_{v}^{d}$ then $v$ is active with respect to $d$, because packets from $w$ to every vertex in $D_{v}^{d}$ travel together with $\langle p\rangle$ until they reach $v$ (the paths are monotone). A vertex which is not active with respect to $d$ is called passive with respect to $d$. Clearly a vertex passive with respect to $d$ must have already received all packets from all vertices in $S_{v}^{d}$. 
Let $k=\log _{2} n$. We will construct a sequence of hypercubes $Q_{0}, \ldots, Q_{n / k}$ such that after moving according to the $i$ th orientation the ratio of passive vertices in $Q_{i}$ with respect to $k i$ is at most $i / 2^{k}$. This would imply that, because if some vertices are active then some packets are not in their destination. For each $i$ the hypercube $Q_{i}$ will have dimension $n-k i$ and it will consist of vertices starting with some string from $\{0,1\}^{k i}$.

Initially all vertices are active and thus we can take $Q_{0}$ to be the whole hypercube. Now we show how to construct $Q_{i+1}$ from $Q_{i}$. Let $Q_{i}$ be an $(n-$ $\mathrm{ki}$ )-dimensional sub-hypercube consisting of vertices starting with some string $x \in\{0,1\}^{k i}$ and suppose that the network is in state after moving according to the $i$ th orientation. Let $M$ denote the number of passive vertices (from $Q_{i}$ ) with respect to $k i$. For any $w \in$ $\{0,1\}^{n-k(i+1)}$ let $A_{w}$ denote the set of active vertices (from $Q_{i}$ ) with respect to $k i$ such that they end with $w$. Now we move the packets along their paths according to the orientation $D G_{i+1}$. How many vertices from $Q_{i}$ ending with $w$ can be passive with respect to $k(i+1)$ after the movement? Let $N_{w}$ denote the set of these vertices. Let $l \in[k]$ be such that $2^{l} \geqslant\left|N_{w}\right|>2^{l-1}$ (the case $\left|N_{w}\right| \leqslant 1$ is treated separately). By induction it is easy to show that there exist distinct $z_{1}, \ldots, z_{l} \in[k]$ such that for each $z_{j}, j \in[l]$, there exist two vertices in $N_{w}$ of the form:


where $a, b, c$ are some binary strings. If there were two vertices in $A_{w}$ of the form:

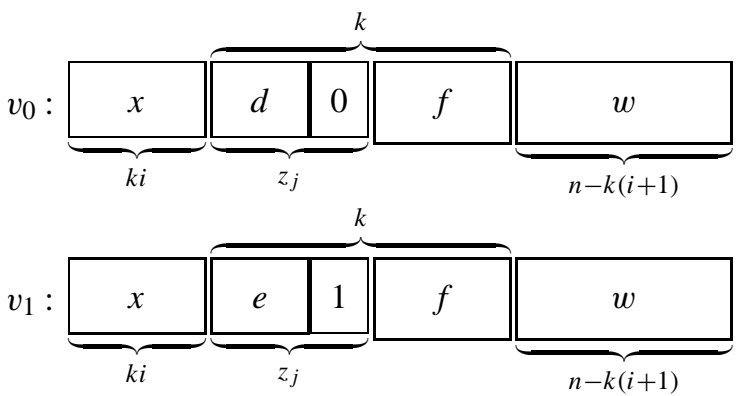

where $d, e, f$ are some binary strings, then either a packet from $v_{0}$ to $u_{1}$ or a packet from $v_{1}$ to $u_{0}$ cannot move between source and destination in the orientation $D G_{i+1}$, because these two packets need to cross the edge between vertices:

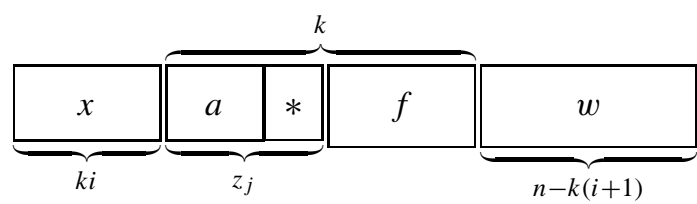

in the opposite direction. This is a contradiction to our assumption that $u_{0}$ and $u_{1}$ will be passive after the movement and thus there cannot be such $v_{0}, v_{1}$ pair in $A_{w}$. Therefore for each $j \in[l]$ the $\left(k i+z_{j}\right)$ th bit of each vertex from $A_{w}$ is determined by bits $(k i+$ $\left.z_{j}+1\right), \ldots, k(i+1)$. This implies that $\left|A_{w}\right| \leqslant 2^{k-l}$. For any $l \in[k]$ :

$\left|N_{w}\right|-\left(2^{k}-\left|A_{w}\right|\right) \leqslant 2^{l}-2^{k}+2^{k-l} \leqslant 1$.

The inequality holds also in case $\left|N_{w}\right| \leqslant 1$. Summing the inequalities for each $w \in\{0,1\}^{n-k(i+1)}$ we obtain:

$N-M \leqslant 2^{n-k(i+1)}$,

where $N$ is the total number of vertices from $Q_{i}$ passive with respect to $k(i+1)$ after the movement. Clearly there exists a string $q \in\{0,1\}^{k}$ such that at most $\left(M+2^{n-k(i+1)}\right) / 2^{k}$ vertices starting with $x q$ are passive with respect to $k(i+1)$ after the movement. Let $Q_{i+1}$ be the hypercube consisting of vertices starting with $x q$. The ratio of passive vertices increased from

$$
\begin{aligned}
& R_{i}=\frac{M}{2^{n-k i}} \text { to } \\
& R_{i+1}=\frac{\left(M+2^{n-k(i+1)}\right) / 2^{k}}{2^{n-k(i+1)}}=R_{i}+\frac{1}{2^{k}} .
\end{aligned}
$$

Therefore if in $Q_{i}$ the ratio was at most $i / 2^{k}$ then in $Q_{i+1}$ the ratio will be at most $(i+1) / 2^{k}$.

Theorem 5. Any shortest-path deadlock-free packet routing algorithm of the graph $G_{n}$ based on acyclic orientations requires $\Omega(n / \log n)$ buffers.

Proof. Let $S$ be the set of all vertices from $G_{n}$ having the head on the leftmost bit and $D$ be the set of all vertices having the head on the rightmost bit. For every $u \in S$ and $v \in D$ there is a unique shortest path from 
$u$ to $v$ in $G_{n}$. It corresponds to changing the bits in which $u$ and $v$ differ while moving the head to the right. Let $\mathcal{P}$ be the set of the shortest paths from every $u \in S$ to every $v \in D$. Let $C=D G_{1}, \ldots, D G_{s}$ be an acyclic orientation cover used to implement shortestpath deadlock-free packet routing algorithm on $G_{n}$. Clearly $C$ is also an acyclic orientation cover for $\mathcal{P}$.

By merging the vertices having the same tape content we obtain the $n$-dimensional hypercube and the path system $\mathcal{P}$ becomes all-to-all path system of the $n$-dimensional hypercube with monotone paths. The acyclic orientation cover $C$ becomes orientation cover of the new path system. Using Lemma 4 we obtain that the size of $C$ must be $\Omega(n / \log n)$.

A consequence of Theorems 3 and 5 is:

Theorem 6. Graph $G_{n}$ of size $\mathcal{N}=n 2^{n}$ has a deadlock-free shortest-path routing algorithm using $\mathrm{O}(1)$ buffers per node, but each deadlock-free shortestpath routing algorithm based on acyclic orientations requires $\Omega(\log \mathcal{N} / \log \log \mathcal{N})$ buffers per node.

\section{Conclusion}

There are many results on buffer-reservation algorithms for specific topologies, but for general graphs very little is known. It would be interesting to have better upper and lower bounds on the number of buffers required for shortest-path deadlock-free routing on general graphs. Concerning acyclic orientations it would be interesting to know how large the gap between routing algorithms based on acyclic orientations and routing algorithms using only plain buffers can be.

\section{Acknowledgement}

Thanks are due to Ivona Bezáková, Rastislav Královič, Branislav Rovan, Peter Ružička and Richard Tan for helpful discussions and for carefully reading the draft of this paper.

\section{References}

[1] B. Awerbuch, S. Kutten, D. Peleg, Efficient deadlock-free routing, in: Proc. 10th Annual ACM Symposium on Principles of Distributed Computing, 1991, pp. 177-188.

[2] R. Cypher, Minimal, deadlock-free routing in hypercubic and arbitrary networks, in: Proc. 7th IEEE Symposium on Parallel and Distributed Processing, 1995.

[3] R. Cypher, L. Gravano, Requirements for deadlock-free, adaptive packet routing, in: Proc. 14th Annual ACM Symposium on Principles of Distributed Computing, 1992, pp. 25-33.

[4] W.J. Dally, C. Seitz, Deadlock-free message routing in multiprocessor interconnection networks, IEEE Trans. Comput. 36 (5) (1987) 547-553.

[5] J. Duato, Deadlock-free adaptive routing algorithms for multicomputers: Evaluation of a new algorithm, in: Proc. 3rd IEEE Symposium on Parallel and Distributed Processing, 1991.

[6] I.S. Gopal, Prevention of store-and-forward deadlock in computer networks, IEEE Trans. Commun. 33 (12) (1985) 12581264.

[7] K.D. Günther, Prevention of deadlocks in packet-switched data transport systems, IEEE Trans. Commun. 29 (4) (1981) 512 524

[8] P.M. Merlin, P.J. Schweitzer, Deadlock avoidance in store-andforward networks, IEEE Trans. Commun. 28 (3) (1980) 345 354.

[9] G. Tel, Deadlock-free packet routing, in: Introduction to Distributed Algorithms, Cambridge University Press, Cambridge, UK, 1994, Chapter 5.

[10] S. Toueg, K. Steiglitz, Some complexity results in the design of deadlock-free packet switching networks, SIAM J. Comput. 10 (4) (1981) 702-712.

[11] S. Toueg, J.D. Ullman, Deadlock-free packet switching networks, SIAM J. Comput. 10 (3) (1981) 594-611. 